\title{
A comparative double blind study of amoxycillin/clavulanate vs placebo in the prevention of infection after animal bites
}

\author{
P. H. BRAKENBURY \& C. MUWANGA
}

Accident and Emergency Department, Middlesbrough General Hospital, Middlesbrough, England

\section{SUMMARY}

The value of prophylactic antibiotics in the treatment of animal bites is uncertain. A prospective double blind stratified trial was performed comparing a broad spectrum antibiotic amoxycillin/clavulanate with placebo in full thickness animal bite wounds in a series of 185 consecutive patients.

In wounds less than $9 \mathrm{~h}$ old, no significant benefit was found with the antibiotic. In older wounds, presenting 9-24 h after injury, the infection rate was reduced significantly $(P=0.023)$, although the numbers were small. We recommend the use of such an antibiotic on wounds $9-24 \mathrm{~h}$ old and query its use in more recent injuries, unless tendons or joints are likely to be involved.

\section{INTRODUCTION}

The main clinical problem a doctor faces in managing patients who present with animal bites, is the prevention of wound infection. The morbidity from such infection can be especially disabling in hand wounds. The magnitude of the problem is shown by the frequency with which such patients attend hospital. In 1986, 1,334 patients with animal bites attended this Department, an incidence of 432 per 100,000 population. This compares with the estimated 300 to 700 animal bites per 100,000 in the USA, Strassburg et al., (1981). The rate of infection has been reported as $4 \cdot 3 \%$ for dog bites and as high as $30-50 \%$ for bites involving the hand (Callaham, 1980; Elenbaus et al., 1982).

Correspondence: Mr P. H. Brakenbury, Accident and Emergency Department, Middlesbrough General Hospital, Ayresome Green Lane, Middlesbrough, Cleveland TS5 5AZ, England. 
The most controversial issue in preventing such infection is the administration of prophylactic antibiotics to these patients, (Snooks, 1982; Brown, 1985). The need for initial thorough wound toilet is generally accepted, (Rosen, 1985) but clinicians and $\stackrel{\$}{\varrho}$ reported trials conflict on the value of prophylactic antibiotics. In discussing the controversy, Snooks (1982) comments on 'the paucity of published studies on this topic $\stackrel{\vec{F}}{\stackrel{9}{+}}$ shows that insufficient attention is given to the review of the more common conditions.'

The aim of this study was to determine the value of amoxycillin/clavulanate $\frac{\bar{\sigma}}{\bar{\omega}}$. (Augmentin $^{\circledR}$ ) for the prophylaxis of wound infection following animal bites, in a㐫 double blind controlled placebo trial. Amoxycillin/clavulanate was chosen as it has a® broad spectrum of antibiotic activity, including pathogens typically associated with $\vec{\circ}$ these wounds such as Pasteurella multicoda, Staphylococcus aureus and anaerobes, $\stackrel{\circ}{\rightarrow}$ (Goldstein et al., 1984). The study received the approval of the Hospital Ethics $\vec{\sigma}$ Committee.

\section{PATIENTS AND METHODS}

Consecutive patients, aged 6 years and above, who presented with full skin thickness $\vec{\nabla}$ animal bites and gave informed consent, were entered into the study. The following cases were excluded-tendon or joint involvement, presentation more than $24 \mathrm{~h}$ after 3 injury and the normal contra-indications to the use of this drug.

On entry into the trial, an initial proforma was completed recording the details of the $\vec{\theta}$ wound. Bacteriological swabs were taken for culture and sensitivity testing. Two bacteriology laboratories were involved, standard mediums being used for aerobic atio anaerobic cultures. One laboratory used blood agar, blood agar with Kanamycin supplement, cysteine lactose electrolyte deficient agar and enrichment culture. The other laboratory used two blood agars, one for aerobic and another for anaerobic $\frac{\circ}{\mathbb{Q}}$ cultures, with McConchie and cooked meat media for subculture. Both laboratories $\stackrel{2}{\vec{B}}$ used the AP1 systems to assist identification of the bacteria.

The initial treatment consisted of thorough wound cleansing with Cetavalon/ Hibitane mixture (Ceticlens), debridement and irrigation with saline as indicated and a? plain tulle dressing applied. Patients were allocated to treatment on a double blind basis $\frac{\Phi}{3}$ using a pre-determined stratified randomization code (one code for hand and wrist:wounds and one for wounds on other parts of the body). Patients aged 6-12 years were 3 . supplied with either Augmentin junior suspension containing $125 \mathrm{mg}$ amoxycillin and $\delta$ $62 \mathrm{mg}$ clavulanic acid or matched placebo suspension and were instructed to take $5 \mathrm{mls}$ tds for 5 days. Patients aged 13 years and over were supplied with Augmentin tablets each consisting of $250 \mathrm{mg}$ amoxycillin and $125 \mathrm{mg}$ clavulanic acid or matched placebo $\frac{7}{2}$ tablets and were instructed to take one tablet tds for 5 days. Follow-up was on day 3 . and, if the wound had not healed, on day 7 or until healing had occurred. Detailed o proformas were completed on each attendance. Healing was defined as occurring when ${ }^{N}$ the skin was no longer broken. If the wound appeared infected, further bacteriological $N$ swabs were taken. Infection was defined as the presence of erythema and tenderness?

\footnotetext{
${ }^{\text {r }}$ Registered trademark of Beecham Pharmaceuticals
} 
beyond that expected $24 \mathrm{~h}$ after the injury with or without purulent discharge, cellulitis or lymphangitis, (Callaham, 1980).

In analyzing the results, the $\chi^{2}$ test was the statistical method used, modified by Yates's correction as required. Fischer's exact test was used in Table 3.

\section{RESULTS}

There were 185 patients entered into the study consisting of 122 adults and 63 children, 123 were male and 62 female. They were divided into four sub-groups: 66 were adults with general bites, 56 were adults with hand bites, 50 were children with general bites and 13 were children with hand bites. The groups were comparable with regard to demographic details as summarized in Table 1 . The bites were caused by dogs in 172 patients (84 amoxycillin/clavulanate and 88 placebo), humans in 8 , cats in 3,1 rabbit and 1 ferret.

Follow-up attendance was $77 \%$ on day 3 and of those requested to reattend $84 \%$ on day 7.

With amoxycillin/clavulanate there was a trend to quicker healing, but there was no significant difference demonstrated between the two treatment groups (Table 2). This trend was also apparent to both patients with hand and general bites. Further analytic breakdown by age of patients, that is children, adults $<50$ years of age, adults $>50$ years of age and by age of wound at entry showed no statistical significant difference in the rate of healing between these sub-groups.

In adults, infection occurred in 17 out of 51 (33\%) receiving amoxycillin/clavulanate and 27 out of $45(60 \%)$ receiving the placebo. This difference in the infection rate for adults is statistically significant $(P=0.009)$. The incidence in children was 7 out of 29 $(24 \%)$ on antibiotic and 5 out of $25(20 \%)$ on placebo.

Table 1 Comparability of groups

\begin{tabular}{|c|c|c|c|c|c|c|}
\hline \multirow{3}{*}{ Drugs: } & \multicolumn{3}{|c|}{ General bites } & \multicolumn{3}{|c|}{ Hand bites } \\
\hline & \multicolumn{2}{|c|}{ Sex } & \multirow{2}{*}{$\begin{array}{c}\text { Mean } \\
\text { Age (years) }\end{array}$} & \multicolumn{2}{|c|}{ Sex } & \multirow{2}{*}{$\begin{array}{c}\text { Mean } \\
\text { Age (years) }\end{array}$} \\
\hline & $\mathbf{M}$ & $\mathbf{F}$ & & $\mathbf{M}$ & $\mathbf{F}$ & \\
\hline \multicolumn{7}{|l|}{ Adults } \\
\hline Amoxycillin/clavulanate & 22 & 10 & $30(n=32)$ & 15 & 10 & $30(n=25)$ \\
\hline Placebo & 22 & 12 & $34(n=34)$ & 21 & 10 & $37(n=31)$ \\
\hline & \multicolumn{3}{|c|}{$\mathrm{N} / \mathrm{S}(P 0.05)$} & \multicolumn{2}{|c|}{$\mathbf{N} / \mathbf{S}(P 0.05)$} & \\
\hline \multicolumn{7}{|l|}{ Children } \\
\hline Amoxycillin/clavulanate & 13 & 12 & $9(n=25)$ & 4 & 2 & $8(n=6)$ \\
\hline \multirow[t]{2}{*}{ Placebo } & 22 & 3 & $9(n=25)$ & 4 & 3 & $10(n=7)$ \\
\hline & \multicolumn{3}{|c|}{$\mathrm{N} / \mathrm{S}(\boldsymbol{P} 0.05)$} & & & \\
\hline
\end{tabular}


Wound infection was reduced significantly by the antibiotic in older wounds, nine or $\bar{\Xi}$ more hours old at entry ( $P=0.023$, Fischer's exact test), but not in more recent wounds $\frac{0}{2}$ (Table 3). In hands and general body bites and in patients over 50 years of age, the $\frac{\mathbb{}}{\varrho}$ reduction in infective rate with antibiotics did not reach significance. It was noted that in the combined placebo and antibiotic groups, the rate of infection was not increased in $\stackrel{\vec{\rho}}{\rightarrow}$ patients over the age of 50 years compared with the adult group as a whole.

Positive cultures of pathogenic organisms were only obtained in 48 out of 185 patients $\frac{\bar{\sigma}}{\overline{\frac{N}{m}}}$ before treatment.

A total of 55 bacteria were isolated and were equally distributed in both treatment groups as detailed in Table 4. Only Pseudomonas was resistant to amoxycillin $/ \vec{\circ}$ clavulanate. Bacteriological swabs were repeated in 22 of these 48 cases, 10 having received the antibiotic and 12 the placebo. The small number of repeat cultures was due $\omega_{\sigma}$ to most wounds being healed on review. In 6 of the 10 antibiotic treated patients and in $\mathbb{D}$ 8 out of the 12 on placebo, the organism isolated on entry to the trial had been $\frac{3}{6}$. eradicated by day 3. After entry into the trial, infection by subsequently acquired $\rightarrow$ organisms occurred in only two patients in the antibiotic group with either a $S$. aureus of or a Citrobacter sp. and in four patients who received the placebo with either $a_{\circ}$ Pseudomonas, Bacteroides or an unidentified Gram-negative rod.

A total of 27 adverse drug reactions were reported in 19 patients, 14 of whom had received amoxycillin/clavulanate acid and five the placebo. All reactions were mild and $\bigodot_{\mathbb{D}}$ none led to withdrawal from the study. The commonest reaction was a gastrointestinal $\frac{3}{0}$ upset present in 10 patients on antibiotics and in one on the placebo.

Table 2 Total number of patients cured by day 7 (cumulative of day $3 \& 7$ assessments) by age of wound $\frac{0}{\mathrm{f}}$ presentation

\begin{tabular}{|c|c|c|c|c|c|}
\hline \multirow[t]{2}{*}{ Treatment } & \multirow[t]{2}{*}{ Number assessed } & \multirow[t]{2}{*}{$\begin{array}{c}\text { Total number } \\
\text { healed }\end{array}$} & \multicolumn{3}{|c|}{$\begin{array}{l}\text { Age of wound at } \\
\text { presentation }\end{array}$} \\
\hline & & & $<2 \mathrm{~h}$ & $2-8 h$ & $8-24 \mathrm{~h}$ \\
\hline Amoxycillin/clavulanate & 75 & 63 & 45 & 11 & 7 \\
\hline Placebo & 68 & 49 & 35 & 7 & 7 \\
\hline Total & $\begin{array}{c}143 \\
\mathrm{~N} / \mathrm{S}(P \quad 0.05)\end{array}$ & 112 & 80 & 18 & 14 \\
\hline
\end{tabular}

Table 3 Incidence of infection associated with wound age at presentation

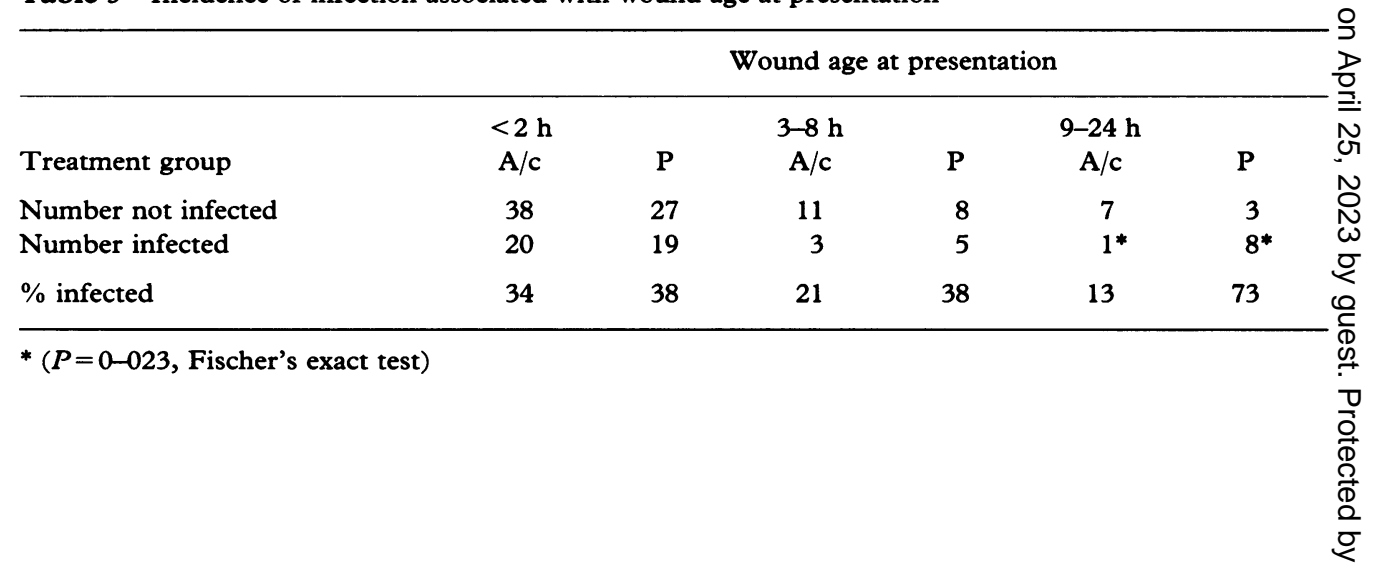


Table 4 Bacteria cultured on entry to study

\begin{tabular}{lcc}
\hline Bacteria isolated & Amoxycillin/Clavulanate & Placebo \\
\hline Acinetobacter sp. & 6 & 10 \\
E. Coli & 6 & 2 \\
Enterobacteriaceae & 2 & 5 \\
Gram-negative rods & 0 & 2 \\
Pasteurella sp. & 1 & 3 \\
Serratia sp. & 1 & 0 \\
Pseudomonas sp. & $1^{*}$ & $1^{*}$ \\
Staphylococcus aureus & 4 & 1 \\
Streptococcus sp. & 4 & 2 \\
Alcaligenes sp. & 0 & 1 \\
Clostridium sp. & 1 & 1 \\
Mixed faecal flora & 1 & 0 \\
\hline
\end{tabular}

* Resistant to amoxycillin/clavulanate

\section{DISCUSSION}

The important finding of this study which contrasts with previous trials, is that an antibiotic, amoxycillin/clavulanate, has been shown to have significantly reduced the wound infection rate in patients with bites where the skin is broken and where the patient presented 9-24 $\mathrm{h}$ after injury. However, there was no significant reduction in the incidence of wound infection for more recent wounds, that is those less than $9 \mathrm{~h}$ old. In earlier trials, the results show no more than a trend towards less infection and faster healing times in those patients receiving antibiotics compared with those receiving a placebo, (Brown et al., 1985). Support for the widespread use of prophylactic antibiotics in treating animal bites has been, until the present time, based on trials which have been criticized for their small size or poor follow-up (Brown et al., 1985). Whilst, in general, these factors were not a problem in this study, a larger group than the 19 patients presenting 9-24 h after injury would be desirable and a follow-up study would be helpful to confirm our findings. In previous papers, the need has been pointed out for a stratified study looking at certain groups of bites which may be particularly at risk (Brown et al., 1985). In this study, three of the factors where prophylactic antibiotic treatment may be of benefit were examined, that is hand bites (Rosen, 1985), patients above the age of 50 years and those presenting late for treatment (Hawkins et al., 1983). We have not confirmed that patients aged over 50 years and those with hand bites were more likely to become infected or to significantly benefit from amoxycillin/clavulanate. It is only in those presenting late for treatment that we have demonstrated a benefit in using antibiotics. It may be argued that the animal bites presenting so late are already in the earliest stages of infection, although not clinically manifested and the use of such antibiotics is not so much prophylaxis as treatment of that infection. It is possible that this may explain why bites presenting earlier benefited less from the antibiotic. Amoxycillin/clavulanate appears to be a particularly suitable broad spectrum antibiotic for the range of bacteria cultured (Goldstein et al., 1984). With only two of the 55 
organisms isolated at entry, both strains of Pseudomonas sp. showing resistance toळ Augmentin.

Minor adverse reactions occurred in 14 out of 88 patients on amoxycillin/clavulanate $\frac{3}{0}$ which were more frequent than in the placebo group, (5 out of 97).

This trial excluded hand wounds involving tendons and joints. It has been argued $\stackrel{\vec{F}}{\rightarrow}$ that such a prophylactic antibiotic should be given in hand wounds (Rosen, 1985) where응 it is difficult to exclude tendon or joint damage. It is our practice that if such structures $\frac{\bar{\sigma}}{\frac{0}{5}}$ are likely to be involved, a broad spectrum antibiotic is prescribed.

We therefore conclude that in the majority of animal bites, presenting early, prophylactic broad spectrum antibiotic treatment offers no significant benefit, although $\vec{\circ}$

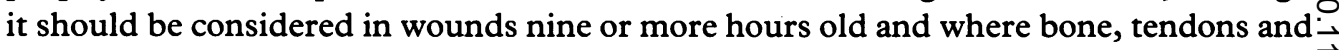
joint involvement is suspected.

\section{ACKNOWLEDGEMENTS}

We wish to express our gratitude to Beecham Research Laboratories for their sponsor- $\stackrel{\text { 을 }}{-}$ ship of this trial and their help with the statistical analysis of the results.

\section{REFERENCES}

Brown C. G \& Ashton J. J. (1985) Dog bites: the controversy continues. American fournal of Emergetie Medicine 3, 83-4.

Callaham M. (1980a) Prophylactic antibiotics in common dog bite wounds: a controlled study. Annals of Emergency Medicine 9, 410-4.

Elenbaas R. M., McNabney W. K. \& Robinson W. A. (1982). Prophylactic oxacillin in dog bite wounds. $\stackrel{\otimes}{\varnothing}$ Annals of Emergency Medicine 11, 248-51.

Goldstein E. J. C., Reinhardt J. R., Murray P. M. \& Finegold S. M. (1984) Animal and human bite wounds: a $\frac{\bar{O}}{3}$ comparative study, Augmentin vs penicillin +/-dicloxacillin. Postgraduate Medicine (Augmentin Symposium Supplement), 105-10.

Hawkins J., Paris P. M. \& Stewart R. D. (1983) Mammalian bites: rational approach to management. Postgraduate Medicine 73, 52-64.

Rosen R. A. (1985) The use of antibiotics in the initial management of recent dog bite wounds. American fournal of Emergency Medicine 3, 19-23.

Snook R. (1982) Dog bites in man. British Medical fournal 284, 293-4.

Strassburg M. A., Greenland S., Marron J. A. \& Mahoney L. E. (1981) Animal bites: patients of treatment. Annals of Emergency Medicine 10, 193-7. 